\title{
Hour Glass Compression Suture in the Management of Primary PPH
}

\author{
Dr. Debjyoti Santra ${ }^{1}$, Dr. Sumanta Kumar Mondal $^{2}$, Dr. Debmalya Maity ${ }^{2}$, \\ Dr. Debasis Das ${ }^{3}$, Dr. Utpal Ghosh ${ }^{4}$ \\ ${ }^{1}$ (Associate Professor, Dept. of Gynaecology and Obstetrics, Bankura Sammilani Medical College , Bankura, \\ India ) \\ ${ }_{2}^{2}(R M O$, Dept. of Gynaecology and Obstetrics, NRS Medical College, Kolkata, India ) \\ ${ }_{3}^{3}$ (Associate Professor, Dept of Community Medicine ,Malda Medical college, Malda, India) \\ ${ }^{4}$ (PGT, Dept. of Gynaecology and Obstetrics, Bankura Sammilani Medical Collegel, Bankura, India,)
}

\begin{abstract}
:
Objective: The present study was planned to access the efficacy of Hour Glass compression suture which is easy, can be applied faster and avoids the need of hysterectomy in patients with atonic postpartum hemorrhage.

Materials and methods: This observational study was carried out from Jan 2010 to October 2012 at Gynaecology and Obstetrics Departments, Bankura Sammilani Medical College \&Hospitals, West Bengal, India. All patients included in this study with primary PPH, hour-glass suture were given using No. 1 Chromic Cat gut with a straight needle. Two suture in a shape of hour glass( figure of 8) on either side of uterus were given, 10 patients presented by severe atonic $P P H$. In all 10 patient, routine initial measures (uterine massage, oxytocin infusion, methergine, carboprost, if not contraindicated, and misoprostol) were done and when failed, compressing uterine suture was performed.
\end{abstract}

Results: All 10 cases had an uneventful postoperative recovery. All the cases had evidence of uterine atony which was not responded to conventional uterotonics. The estimated blood loss ranged from $1000 \mathrm{ml}$ to 2,000 $\mathrm{ml}$. In all cases this technique successfully stopped the bleeding and the patient's uterus was preserved. No complication like pyometra, amenorrahoea/oligomenorrahoea, suture cut down through myometrium was faced during the procedure. Regular menstruation returned within 3-4 months follow up after operation, except in one case where the patients conceived during the period of lactational amenorrhoea. Later she opted for medical termination of pregnancy.None of them require hysterectomy. Conclusion: The technique is easy, rapid and requires less skill and this simple procedure be tried first before other complex measures like uterine artery ,ovarian artery ligations are undertaken particularly for those obstetricians who lack sufficient training and skill.

Key Words: Hour Glass compression suture, Postpartum hemorrhage, Hysterectomy.

\section{Introduction}

It has been estimated that nearly 1, 25,000 mothers die of PPH worldwide each year ${ }^{1}$, contributing 25 $43 \%$ of all maternal death in the developing country. Primary PPH occurs within 24 hour of delivery. It is defined as a reduction in the patient's haematocrit level of more than $10 \%$ of the prenatal value or blood loss causing hemodynamic instability of sufficient seriousness to require blood transfusion. (ACOG1998). Alternatively $>500 \mathrm{ml}$ blood loss in vaginal delivery $\&>1000 \mathrm{ml}$ blood loss in caesarean section considered as an upper border of normal limit. Nearly $45 \%$ of post-partum death occur in the first $24 \mathrm{hr}$ after delivery ${ }^{2}$.Uterine atony accounts for $75-90 \%$ of primary PPH. It is mainly controlled by bimanual uterine compression \& uterotonic agents. However many cases of atonic uterus may not respond to above management, particularly in cases of prolonged and augmented labour with an infected and exhausted uterus. In that case various surgical techniques are necessary including uterine tamponade with packing, uterine compression sutures, selective ligation of major vessels and hysterectomy as last resort.

In recent decades, active attempts have been made to introduce conservative procedures to avoid hysterectomy when uterotonic medications failed to control bleeding in massive PPH. particularly, within the past years, interest has surged in the surgical compression sutures for treating PPH due to uterine atony, by exerting a mechanical compression of uterine vascular sinus without occluding either the uterine arteries or the uterine cavity. Of the several different techniques, the B-Lynch suture ${ }^{3}$, first reported in 1997 by Christopher B Lynch, has gained the most popularity. 
B-Lynch suturing technique is particularly useful due to its simplicity of application, life saving potential, safety and capacity for preserving the uterus and subsequent fertility ${ }^{7}$.In recent review Holtsema et al argued that the B-Lynch technique for postpartum haemorrhage should be an option for every obstetrician ${ }^{8}$

Since its introduction several modification of the original technique was made \& published in the literature. Some popular modifications were made by Cho et al (2000), Hayman et al (2002), Bhal et al (2005) Nelson \& Birch (2006) etc.

However, theoretical concerns have been raised on the potential risk of occlusion of the uterine cavity and blood entrapment, as the uterus is transfixed from front to back to place the suture ${ }^{1,4}$. Since the technique is relatively new, data on its safety and efficacy are limited to a few case reports ${ }^{3,5}$.

In the present study we described our experience over the last two year in using hour glass suture as an alternative of the conventional compression suture to treat primary PPH

\section{Materials And Methods}

This observational study was carried out at Gynaecology and Obstetrics Departments, Bankura Sammilani Medical College \&Hospitals, West Bengal, India .All patients included in this study with primary PPH, hour-glass suture were given using No. 1 Chromic Cat gut with a straight needle. Two suture in a shape of hour glass( figure of 8 ) on either side of uterus were given, 10 patients presented by severe atonic PPH in the period from Jan 2010 to October 2012. In all 10 patient, routine initial measures (uterine massage, oxytocin infusion, methergine, carboprost ,if not contraindicated, and misoprostol) were done and when failed, compressing uterine suture was performed. The need for a hysterectomy was avoided in all cases. This compression technique was not supported by other major vessel (uterine, ovarian etc) ligation technique. The suture was given using no. 1 chromic catgut. The proper steps and method is described by the schematic diagram below. The cases were followed up from the day of operation till October 2012.

\section{Description of the Hour glass suture Technique.}

Uniform protocol for the management of obstetrics haemorrhage were in use in our institution. Prophylactic oxytocis were offered routinely in the management of the third stage of labour. Once PPH has been identified and after vaginal/cervical laceration or hematoma and retained product of conceptus have been ruled out, following measures were used initially to combat the bleeding-infusion of oxytocin ( 20 iu in $500 \mathrm{ml}$ of normal saline@125ml/hr) ,methyl ergometrine $0.2 \mathrm{mg}$ intramuscularly, carboprost tromethamine $250 \mu \mathrm{g}$ intramuscularly, $800 \mu \mathrm{g}$ misoprostol given per-rectally.In all the above cases medical management failed to controlled the haemorrhage and continued to bleed, haemodynamically unstable ,surgical haemostasis was started. Uterus was exteriorised, bimanual compression was applied to check whether this method stopped the bleeding, before surgical suture was given.

\section{Procedure:}

(1) The patient is catheterized ( indwelling foley's) and placed in the Lithotomy position for access to the vagina to assess the control of bleeding

(2) The abdomen is opened by a Pfannenstiel incision in normal vaginal delivery or intraoperatively during caesarean section, or if the patient had caesarean section following which she bled, the same incision is reopened.

(3) a) Intraoperatively during caesarean section

A No 1 chromic cat gut is (threaded with straight needle) passed through the uterus $3 \mathrm{~cm}$ below the right lower margin of the uterine incision and $3 \mathrm{~cm}$ from the right lateral border the uterus through uterine cavity from anterior to posterior, it is then passed through at the middle of the uterus form posterior to anterior .the suture (now visible) is passed over to compress the uterine fundus approximately $3-4 \mathrm{~cm}$ from the right cornual border. It is then fed through from posterior to anterior (at the same level where the suture is threaded before during posterior to anterior entrance). The two lengths of suture is pulled under moderate tension assisted by bi- manual compression exerted by the first assistant. Meanwhile, the vagina is checked for bleeding. Once haemostasis is secured and uterus is compressed, the two length of the suture is tightened by double knot followed by two or three further knots to secure the tension. The same procedure is carried out on the left side of the uterus with No. 1 cat gut. Lower uterine incision is sutured in two layer.

b) Following normal delivery , the abdomen is opened by a Pfannenstiel incision.

The loose peritoneum of the utero-vesical pouch is cut transversely across the lower segment with convexity downwards at about $1.25 \mathrm{~cm}(1 / 2$ inch) below its firm attachment to the uterus. A No 1 chromic cat gut is (threaded with straight needle) passed through the uterus $3 \mathrm{~cm}$ below the right lower cut margin of the loose peritoneum and $3 \mathrm{~cm}$ from the right lateral border 
of uterus. The remaining procedure is same as followed as in 3 (a) on right as well as left side.

(5) Abdomen closed in layer.

(6) Vagina is re-inspected for any bleeding

\section{Results}

The Hour glass compression suture was attempted in a total 10 cases from January 2010 to October 2012. All 10 cases had an uneventful postoperative recovery. All the cases had evidence of uterine atony which was not responded to conventional uterotonics. The estimated blood loss ranged from $1000 \mathrm{ml}$ to $2,000 \mathrm{ml}$. In all cases this technique successfully stopped the bleeding and the patient's uterus was preserved. Regular menstruation returned within 3-4 months follow up after operation, except in one case where the patients conceived during the period of lactational amenorrhoea. Later she opted for medical termination of pregnancy. In this study population some patient needs special elaboration (details given in table 1). Case no. 3 was of a primi mother who came to our hospital after suffering from agonising labour pain for 3 days, due to obstructed labour at home. Eventually the baby was found dead and she landed up into severe PPH immediately after delivery. Later she responded poorly to conventional medical management and surgical options were only left as the life saving tools. Hour glass compression suture enabled us to preserve the uterus without endangering her life. In case no. 4 was the mother opted for delivery in the primary health centre. She referred to our hospital with acute inversion of uterus due to inadvertent pull in a case of adhered placenta. She was managed by reposition of uterus followed by manual removal and lastly her PPH was controlled by hour glass suture under general anaesthesia. In case no. 6 \& 7 we had to apply three hour glass suture where as only two was adequate for the rest of the cases.

\section{DISCUSSION}

This study was done in a rural tertiary referral centre catering adjacent 5 backward districts with approximate 20,000 annual delivery rates. Major problem faced regarding managements of critical obstetrical cases were delayed referral due to poor transport facility \& lack of awareness among people along with lack of health care personnel. Commonly they suffered from serious obstetrical haemorrhage as a consequence of mismanaged labour at home or in the peripheral centre. Majority of them responded well with conventional medical management. Eventually there were few cases of non responder, whose fertility had been sacrificed to save their life. Due to this background there is a constant demand and scope of improvement in the field of conservative managements of primary PPH.

In this study we had evaluated an Hour glass suturing technique for achieving compression in primary PPH. We found that this procedure was very safe and inexpensive. It was proved to be very effective also, as it was able to prevent difficult peripartum hysterectomy in all cases we observed. It was also less time consuming as mean operating time for the procedure proper (hour glass compression suture) was only $5 \mathrm{~min}$ (4- $6.5 \mathrm{~min}$ ) Compared with the B-Lynch suture, the Hayman technique is a much simpler procedure, which can easily be mastered by every obstetrician in an emergency situation. Moreover, the Hayman suture is quicker to apply in cases of major PPH following vaginal delivery, as the lower uterine segment is not opened. It has been argued that hysterotomy before placement of compression sutures is warranted to avoid unintended obliteration of the cervical canal and/or the uterine cavity and to confirm that the uterine cavity is completely empty ${ }^{4}$. Moreover, since the Hayman technique involves crossing the uterine cavity to appose the anterior and posterior uterine wall above the bladder reflection, theoretical concerns on the potential risks of cavity occlusion and infections have been raised ${ }^{5,6}$.

We also observed that, in comparison with conventional B Lynch and other technique this simple procedure can easily be mastered by every obstetrician working in emergency situation. None of these patients suffered from the documented complication of this procedure like pyometra, uterine necrosis or re-laparotomy. Data regarding menstrual periods after uterine compression suture is limited. Normal menstrual patterns returned in five patients out of seven after having $U$ suture done by Hackelthal et al. Quahba et al. reported that all women recovered normal menstrual cycles. Even in Cho's study all patients recovered normal menstruation. Although there is no reported cases of amenorrhoea or oligomenorrhoea after compression suture , detailed follow up of these patients have not been documented completely. Api from Turkey a case of successful pregnancy after hypogastric artery ligation and B-Lynch suturing technique ${ }^{9}$.Tsitlakidis reported a follow up of a case with successful pregnancy 10 yrs after PPH was managed with B-Lynch suture This case is the longest follow up till now after B-Lynch suture application ${ }^{10}$. Habek Croatia reported a successful term pregnancy in a28 yrs old patient after previous caesarean section and B-Lynch compression suture ${ }^{11}$.

In our study all women returned with normal menstrual cycles. One of the patients in this study group conceived during the period of follow-up, but she opted for medical termination. No complication like pyometra, amenorrahoea/oligomenorrahoea, suture cut down through myometrium was faced during the procedure. 
Major advantage of this technique is that the uterine cavity was not opened during the procedure which save critical time \& reduce the incidence of endometritis or sepsis. To prevent entrapment of retained bits of placenta or blood clot, meticulous evacuation and sustained bimanual pressure was used during the procedure.

There were few drawbacks of this study as it was not randomized and followed up for lesser duration post-operatively. Data \& results should be cautiously interpreted as only 10 cases were included in this study.

\section{Conclusion}

Two parallel sutures( Hour glass sutures) placed in the uterus controls bleeding effectively. The technique is easy, rapid and requires less skill and this simple procedure be tried first before other complex measures like uterine artery, ovarian artery ligations are undertaken particularly for those obstetricians who lack sufficient training and skill.

\section{References:}

[1]. Hayman R G, Arulkumaran S, Steer P J. Uterine compression sutures: Surgical management of postpartum haemorrhage. Obstet Gynecol 2002; 99:502-6

[2]. Li XF ,Fortney JA, Kotelchuck M, Glover LH(1996) The post partum period ; the key to maternal mortality. Int J Gynaecol Obstet 52:1-10

[3]. B-Lynch C, Coker A, Lawal A, Abu J, Cowen M. The B-Lynch surgical technique for the control of massive postpartum haemorrhage: an alternative to hysterectomy? Five cases reported. Br J Obstet Gynecol 1997;104:372-5

[4]. Allam MS, B-Lynch C. The B-Lynch and other uterine compression suture techniques. Int J Gynaecol Obstet 2005;89:236-41

[5]. B-Lynch C. Partial ischemic necrosis of the uterus following a uterine brace compression suture. BJOG 2005;112:126-7.

[6]. Cotzias C, Girling J. Uterine compression suture without hysterotomy-why a non-absorbable suture should be avoided. J Obstet Gynaecol 2005;25:150-2.

[7]. Prince N, B-lynch C(2005) Technical description of the B-Lynch brace suture for treatment of massive post partum haemorrhage and review of published cases.Int. J Fertility Women's Med 50(4):148-163

[8]. Holtsema H,Nijland R, Huisman a,Dony J,van den Berg PP(2004) The B-Lynch technique foe postpartum haemorrhage: an option for every gynaecologist.Eur J Obstet Gynaecol Reproductive Biol 115:39-42

[9]. Api M,Api O, Yayla M (2005) Fertility after B-Lynch suture and hypogatric artery ligation.fertil Steril 84(2):509

[10]. Tsitlakidis C,Alalade A,Danso D, B-Lynch C (2006) Ten year follow of the effect of the B-Lynch uterine compression suture for massive post partum haemorrhage. Int. J Fertility Women's Med 51(6):262-265

[11]. Habek D ,Vranjes M, Bobic Vukobic M, Valetic J, Kremer V,Simunac J (2006) Successful term pregnancy after B-Lynch compression suture in aprevious pregnancy on account of massive primary postpartum haemorrhage. Fetal diagn Ther 21 (5) : 475-476

Table 1. Summary of patient characteristics and clinical outcomes of the use of Hourglass suture for the treatment of atonic PPH

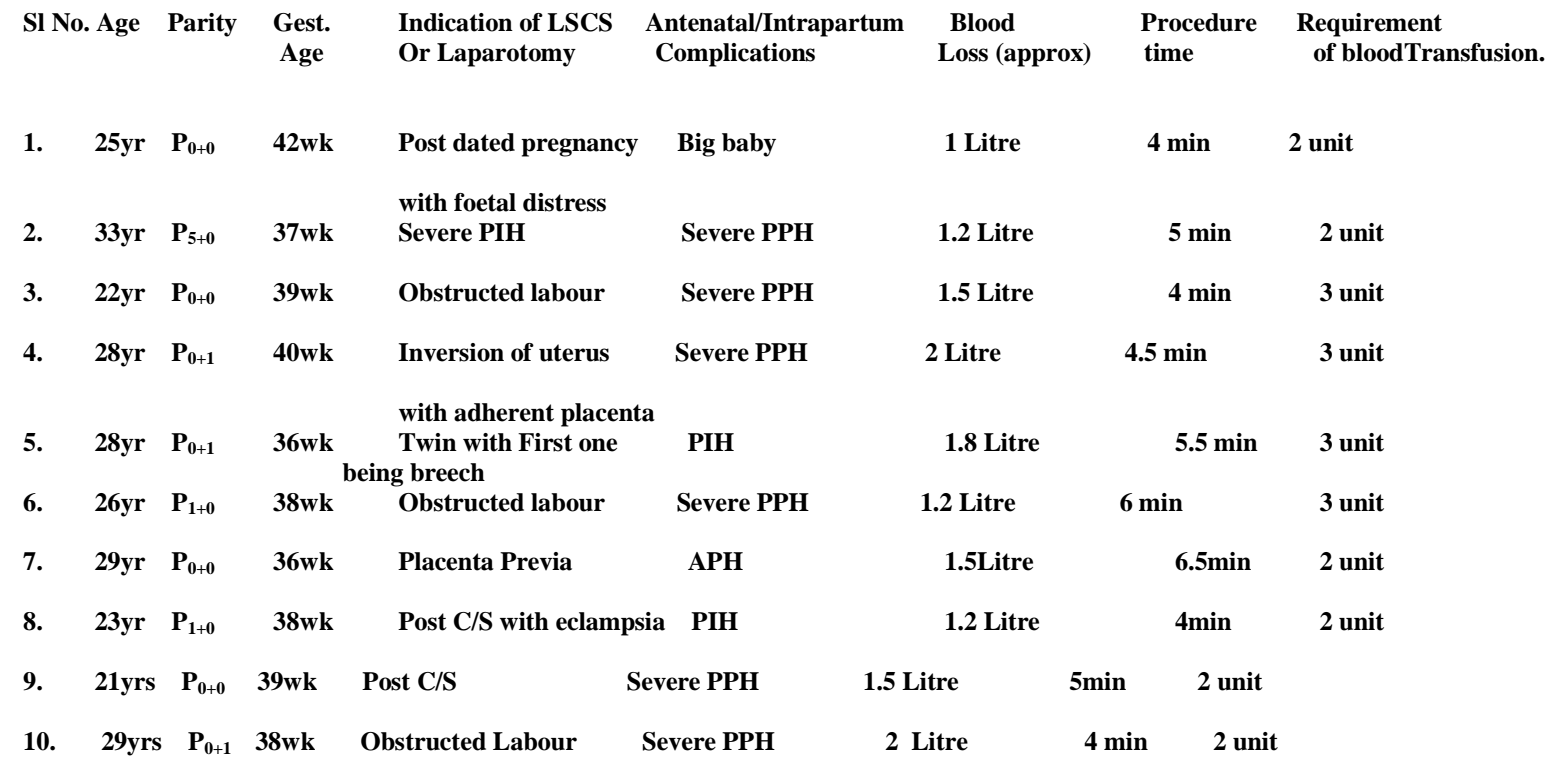


Figure 1.

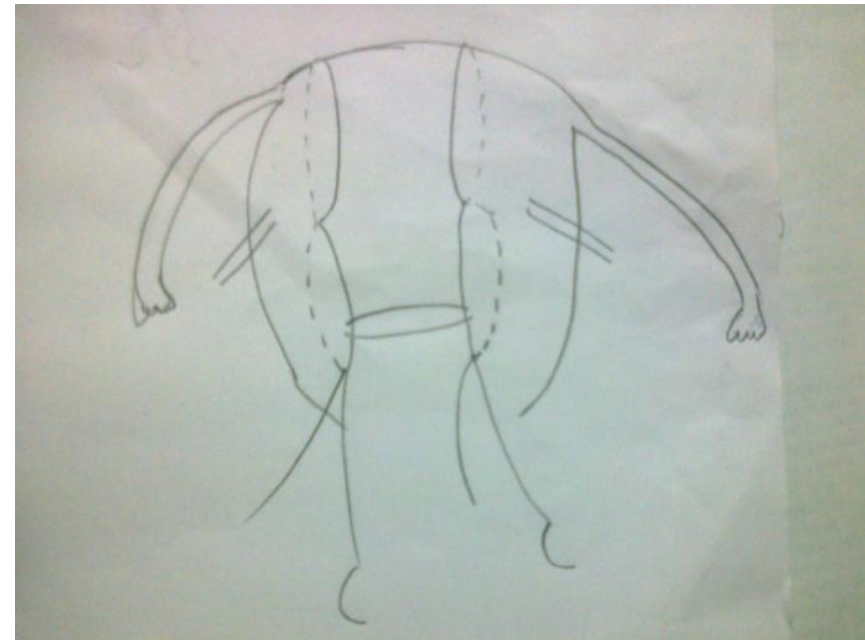

Schematic diagram of Hour glass suture

Figure 2.

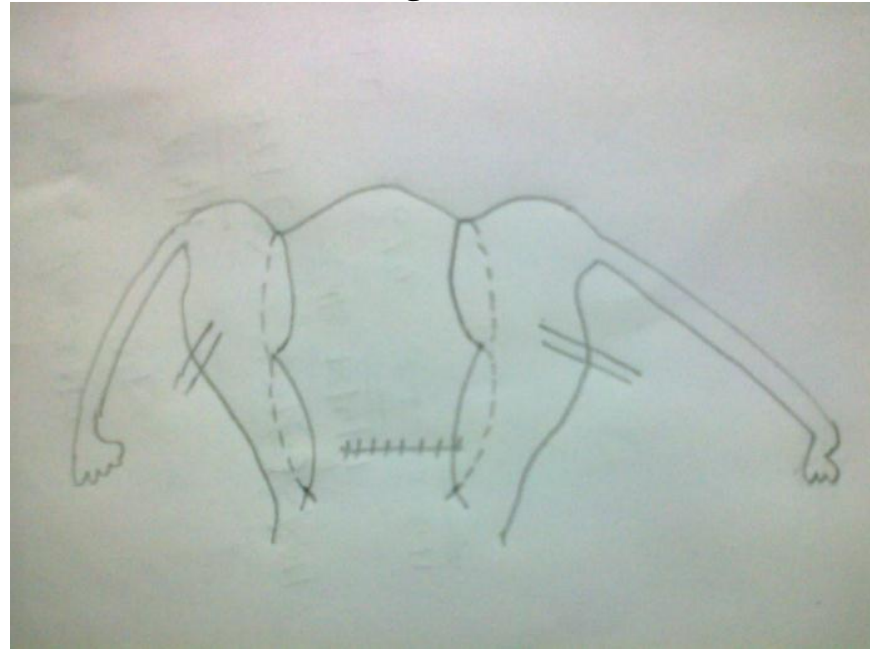

Schematic diagram of Hour glass suture

( After suture tied and uterus compressed with closer of the uterine incision) Figure 3.

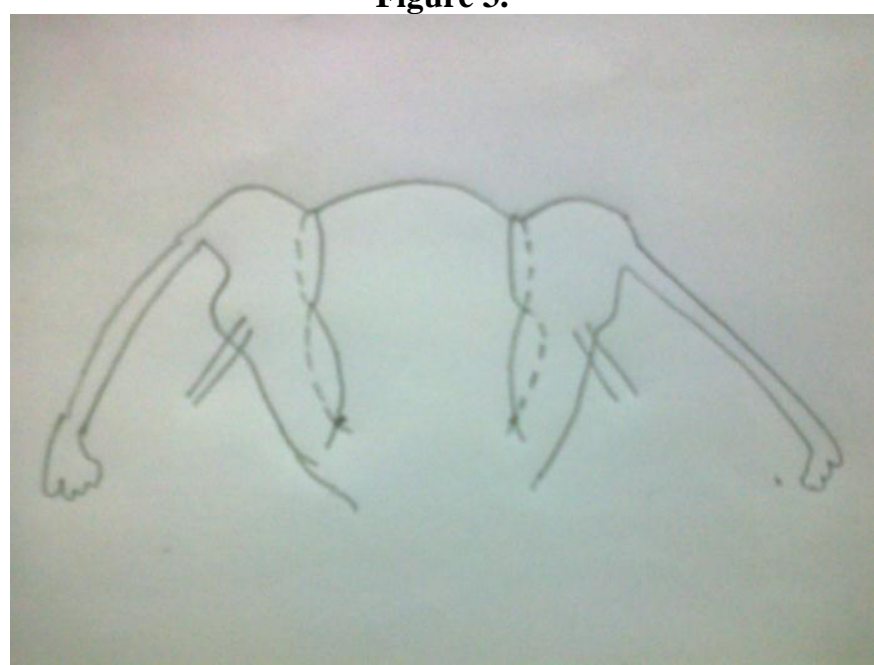

Schematic diagram of Hour glass suture

( After suture tied and uterus compressed where primary Hour glass suture given without opening the uterine cavity) 
Figure 4.

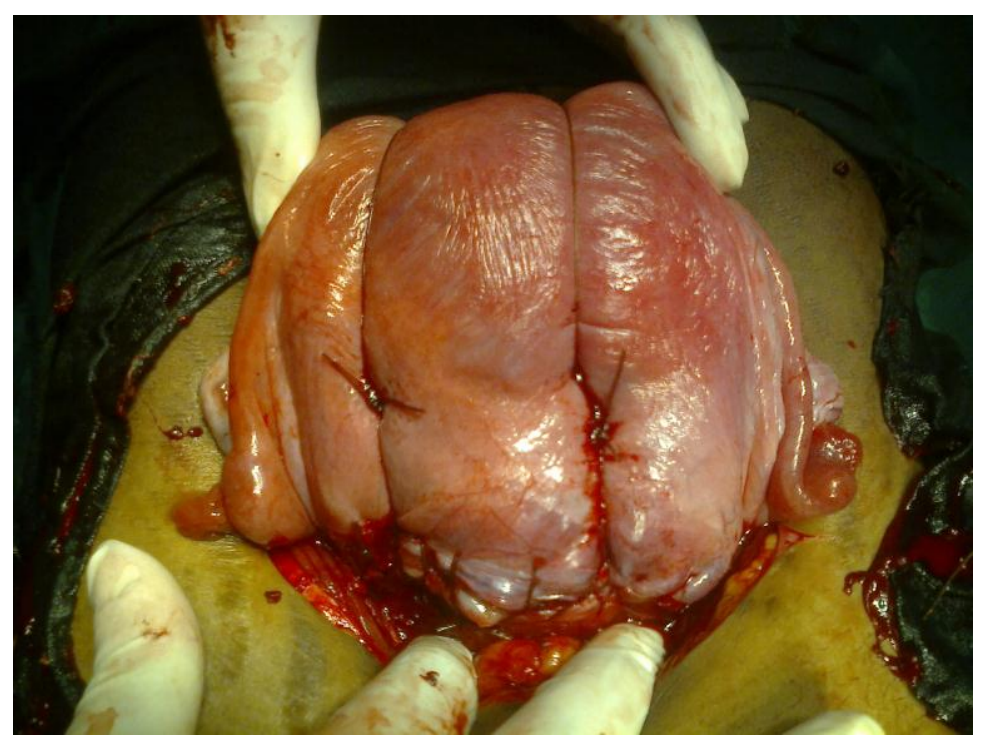

Anterior view of Hour glass suture

Figure 5.

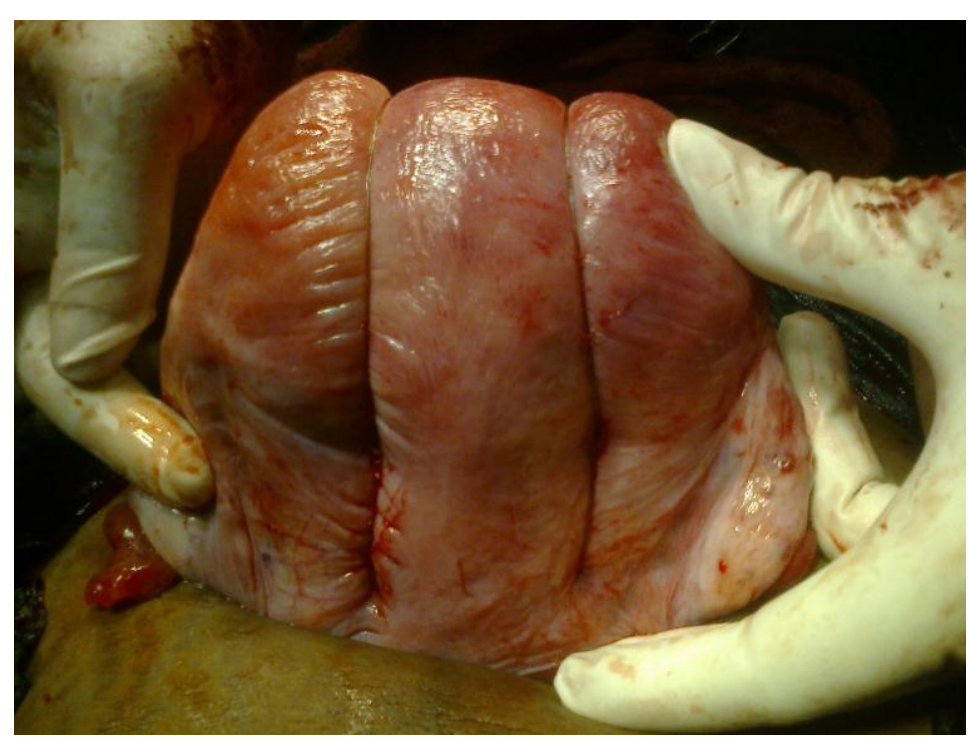

Posterior view of Hour glass suture 